\title{
The trade-off: a central concept for biomimetics
}

Julian F.V. Vincent MA, PhD, DSc, MIM, CEng, FIMechE*

Senior Research Associate, Department of Zoology, University of Oxford,

Oxford, UK

Biology is gradually yielding lessons and ideas for technology, but the resulting innovation is adventitious. Biology is also very complex: currently with no underlying analytical model and so cannot adequately be interrogated by technologists. A concept which can bridge this gap is the trade-off, which leads to speciation in biology and aspects of design and problem-solving in engineering. An ontology is described which uses biological organisms as case studies. Terms have been adapted from TRIZ (the Russian system for solving problems creatively) to define trade-offs and the factors by which they can be manipulated. As an example the ontology resolves the biological trade-off "speed-accuracy", yielding factors for a biomimetic version of the trade-off. These are Feedback (e.g. errorcorrection), Dynamic Response (e.g. control of thresholds), Adaptation (e.g. ability to predict) and Consolidation (e.g. stochastic accumulation). Since much of evolution can be related to the resolution of trade-offs, it is expected that this ontology can also categorise aspects of natural selection. Multi-criteria analysis will allow more versatile numerical models to be developed.

\section{Introduction}

Evolution occurs both in biology and in technology. ${ }^{1}$ Selective pressure and phylogenetic evolution of biology translate into market pressures and advances in technology, so it is at this level that a bridge for biomimetics can profitably be built. The method is to state a problem in a manner that can be understood, in every way, in the contexts both of biology and of engineering. This addresses one of the big problems in biomimetics, which is that the databases that so many people have produced are about the actions that organisms and engineering perform, not the problems that organisms (by natural selection and evolution) and engineering (by experiment and design) resolve. It is the resolution of those problems that leads to adaptation of organisms and improvements in design and technology. What myriad of problems were solved to give a motorcar, inscrutably moving through the countryside? Just by looking at the car and its parts, in whatever detail, it cannot be known what those problems were. The answers to those problems are being looked at in ignorance of the alternatives that were rejected - for reasons to do with physics, engineering, commerce or tradition - during the development of that motorcar. And the answers that are being looked at pertain to only a subset of the contexts in which that motorcar can thrive. An off-road vehicle will be different from a racing car, although they share a large number of functional subunits. The point of biomimetics is to see how organisms have adapted for survival and to transfer those adaptations, in appropriate form, into a technical context. But unless the environmental and physiological selection pressures can be defined, the adaptations cannot be understood because what is and is not relevant cannot be properly understood. The selection pressures need to be defined and stated in some form which reduces the importance of context. The easiest way to do this seems to be as a trade-off, a term common to both biology and engineering. In the simplest form of trade-off, a dichotomy, one finds the statement 'trade-off between [A] and [B]', where A and $B$ between them define the trade-off. This trade-off can be further categorised as follows.

- Type 1: Both A and B may be preferred, but at different times under different circumstances, so the trade-off becomes subject to external (or internal) conditions and can be an adaptation mechanism.

- Type 2: A and B may be antagonistic, in which case the tradeoff might be spoken of as a compromise or an optimisation.

- Type 3: A and B may be exclusive, in which case there is only one choice, as in gene repression.

\section{The trade-off}

Here is a simple trade-off. ${ }^{2}$ A person wants to buy a car, but he does not have much money, but he would like to have a car which does not release too much carbon dioxide $\left(\mathrm{CO}_{2}\right)$. But the least-polluting cars are rather expensive, and the cheapest cars, even though they have smaller engines, also produce more carbon dioxide per kilometre travelled (Figure 1). So the person compromises. It is a trade-off of type 1 between cost and carbon dioxide, and he takes a middle path. But he does not need to. The line on the graph is a boundary defining the limit of the best available combinations of carbon dioxide output and price. It is called the Pareto front (it is the advancing edge of a population of cars whose cost and carbon dioxide production is plotted, so there 


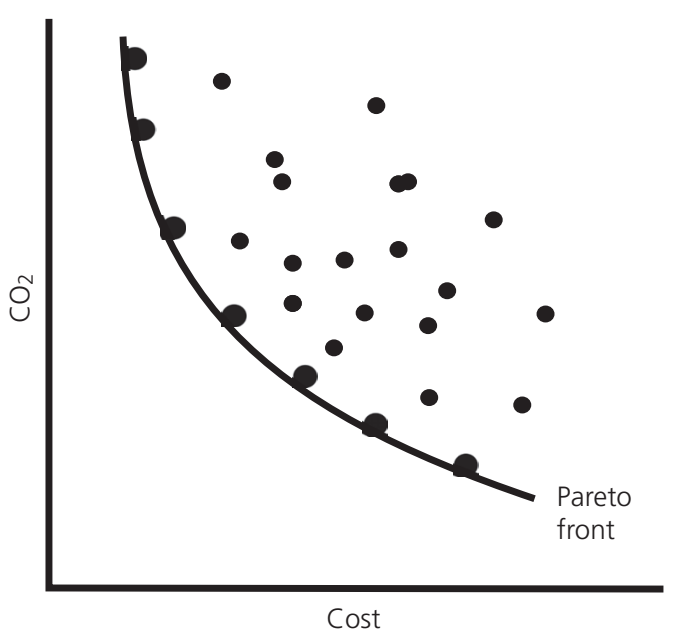

Figure 1. Cost of a car and the amount of carbon dioxide it produces in the exhaust. The line is drawn through points indicating the best choices considering both factors

is nothing in the area below and to the left of this line) or the Pareto set (the points that sit on this Pareto front form a group or set with the common factor of being the best choice for any particular combination of cost and carbon dioxide). In fact, any member of the Pareto set represents a car whose desirability resides in the best combination of its price (and, hence, one hopes, sense of luxury or perhaps of asceticism) and its production of carbon dioxide. Changing the position of the choice by moving along the front represents adaptiveness, an important consideration in biological fitness and success. The position along this curve which is finally chosen is therefore heavily influenced by a context which the graph does not illustrate. Pareto analysis is also called multicriteria analysis or multiobjective analysis. ${ }^{3}$

An example of a trade-off in biology which invokes a large part of the Pareto front is that between reproduction and immunity. In mammals, insects and other animals in which the eggs are fertilised inside the female, the male's sperm enters her body and will be recognised as alien. Under normal circumstances, her immune system would step in and neutralise the invading secretions. But that would defeat the object of reproduction. Instead, the female downgrades her immune system so that a sperm can reach an egg and fertilise it. But this now leaves her open to disease and parasites. ${ }^{4,5}$ So (among humans, at least) she tends to hide out of the way, become torpid and avoids contact with external threats. This trade-off with its control methods (for instance, specific hormones) could give information for clean manufacturing by using a range of materials and structures and adequately describes origination and conditions of quarantine.

One of the big difficulties in understanding the trade-offs that confront organisms, and to which they have to adapt in some way, is that there can be so many potential trade-offs at any one time. In contrast to the simple dichotomy which is often presented, with a mathematical model such as Pareto analysis, there is no conceptual limit on the number of trade-offs which can be considered at any one time. However, the extremely hierarchical structure of biological morphology and processes, both developmental and physiological, imposes an extra constraint in that there will be a cascade of Pareto fronts, each of which will depend on the chosen optimisation of the previous front in the cascade. The net or ultimate trade-off then depends on a number of factors which can be of type 1 or type 2 . Survival in a changing environment is presumably maximised if all trade-offs are type 1 .

The division of one species into two can also be the result of a trade-off, ${ }^{6}$ where there are advantages at either end of the Pareto front. Consider the following scenario. Some aquatic algae tend to float to the surface of their pond during the day and so photosynthesise more. But the fish in the pond can see the algae easily against the clear sky and eat them. The algae sink to the bottom of the pond at night since there is no light near the surface and are safe from the fish, but they are colonised by lethal bacteria. A solution here would be for the algae to sequester the bacteria during the night and pass them on to the fish when they are eaten. The fish might then die, or at least find the algae unpalatable. But another solution might be for the algae to separate off into two populations - the algae at the surface manage to reproduce fast enough to compensate for predation, perhaps by developing thicker cell walls with all that photosynthetic energy. Meanwhile, the algae in the mud have a reduced level of photosynthesis but reduce their level of metabolism and manage to survive with the bacteria. Reproductive isolation follows spatial isolation, and the two populations are on their way to speciation as the result of trade-offs.

In order for a trade-off to function properly as a tool for biomimetics, it helps to put it into a context and map out a network connecting the ideas and observations involved (Figure 2).

\section{Origin of the concept of trade-off}

It may help to know where the idea of trade-off comes from. It is an old idea, popularised by Plato's Socratic dialogues, that in order to establish a truth it is necessary to have two or more people with opposing views to engage in dispassionate discussion until a resolution is reached. ${ }^{7}$ This unity of opposites - the dialectic - was proposed by Heraclitus of Ephesus, who said that everything is in constant change, as a result of inner strife and opposition (unfortunately, Heraclitus never published his ideas formally). Over the years, many forms of dialectic have arisen; the one most familiar to Europeans is the Hegelian dialectic, although Hegel said that he got the idea from Kant. Kant named the two opposites thesis and antithesis, and the resolution he called the synthesis. The synthesis can then become the thesis in a new dialectic. In fact, it serves little use to spend much time on this, in particular since Karl Popper, a philosopher of science in the twentieth century, maintained that advances are really made by trial-and-error searches and that the dialectic is a rationalisation of the outcome. ${ }^{8}$ But, of course, evolution is all about trial and 


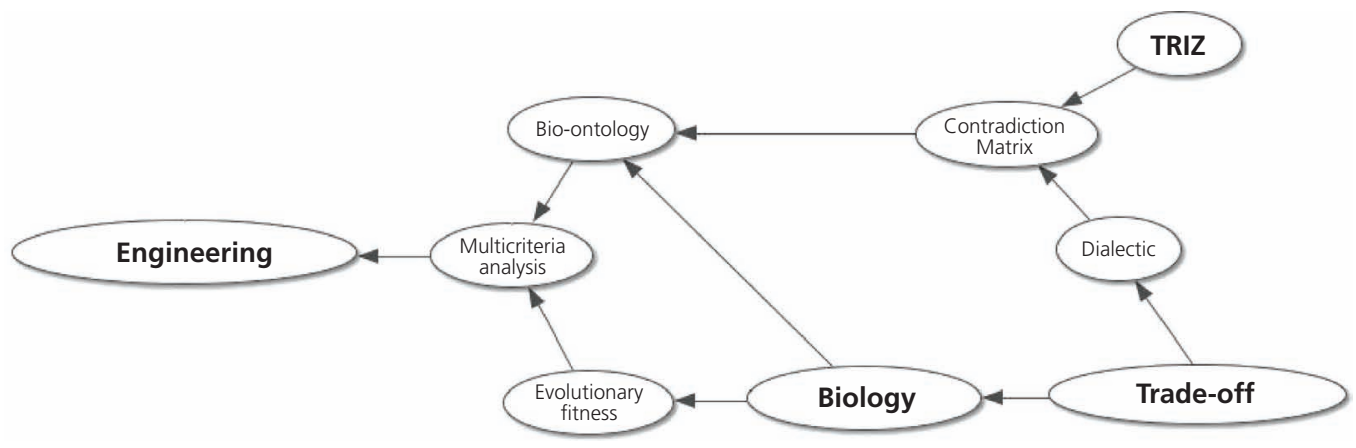

Figure 2. Relationships between the various components contributing to the idea behind the ontology

error. The error is the variation that occurs from generation to generation; the trial leads to the removal of substandard individuals from the population. An important point is that the names 'thesis' and 'antithesis' may suggest 'right' and 'wrong'. The two should be accepted as equal, with no bias towards either being accepted as a solution to the dialectic argument. It may be, and often is, advantageous that the dialectic is not resolved to a unique solution but that the argument leads to better understanding of the variables which allow movement between the thesis and its opposite in response to prevailing selection pressures. For instance, an organism can change its physiology in response to hormonal mediation of environmental changes as when growth or reproduction synchronises with annual changes in temperature and day length.

\section{The trade-off and TRIZ}

The Hegelian concept of the dialectic is (or was) widely taught in continental Europe. Genrich Altshuller, a Russian engineer and inventor from Tashkent, used this concept when developing one of the early techniques of TRIZ $^{9}$ which he called the Contradiction Matrix. In a lengthy study of several thousand patents, Altshuller drew up a list of 39 characteristic factors which are named Engineering Parameters that might affect the performance of an engineering machine, material or structure. From this list, one selects two parameters which, taken together, define the problem under investigation, a process that Heraclitus would have recognised. For instance, a bridge may be called on to take more traffic, but it is not possible to increase the size of the foundations (the 'impossible' problems are probably easier to resolve because the problem is more clearly stated). This can be restated as a trade-off between resisting greater force or changing the supporting shape. (An example where the shape-change option was successful is the bridge over Auckland harbour, where two extra carriageways were added outboard of the existing ones. A Japanese design, this was memorably christened the 'Nippon clip-on'). In this form the problem, now defined in a neutral and objective manner, becomes generalised such that other problems already solved, which can be defined in the same way, can offer strong solutions. These solutions comprise the TRIZ list of recommendations - the Inventive Principles - numbered 1 to $40 .^{10}$ There is nothing formal about the derivation of any of this system, and it is unfortunate that there is no justification or verification for any of the parameters or the principles recorded in any literature. However, up to a point, the system works. The 39 parameters are arranged around two sides of a $39 \times 39$ matrix, those along the side of the matrix defining thesis and those along the top defining antithesis. The matrix therefore defines just over 1500 problems, and the body of the matrix is populated with relevant principles according to the combination of thesis and antithesis intersecting at that point. A matrix with the same items on each of the two axes might be expected to be symmetrical about the diagonal. But the Contradiction Matrix is unusual in that in the format originally conceived, the dimensions represent positive (vertical dimension) and negative (horizontal dimension) factors. Notwithstanding, the matrix reveals a significant degree of symmetry about the diagonal (Figure 3) such that some $27 \%$ of the squares on one side of the diagonal have identical twins on the other side. And a further $23 \%$ have only one principle differing. Thus, the two dimensions of the matrix are significantly interchangeable and the differentiation between thesis and antithesis is clouded. In at least half the cases, it appears that the Inventive Principles are not suggesting ways in which a problem can be solved, so much as how the Pareto front might be navigated. $\mathrm{Deb}^{3}$ reasoned that this will produce a better solution to a problem, since it allows context to be used in making the choice. Deb also pointed out that it is easier to identify the correct parameters in the presence of a number of Pareto-optimal solutions. This makes the choice of solution far less subjective, since in the TRIZ model, the choice of parameter can be subjective and the suggested solution can be very sensitive to this choice. In a pragmatic approach to such sensitivity, the TRIZ protocol suggests choosing a number of closely related parameters to see which principles are most frequently suggested. This is akin to the process detailed in choosing principles for resolving a tradeoff (see below).

The fact that both parameters and principles are expressed in general terms (e.g. 'make it bigger', 'use a cheap copy instead of the original' and 'loss of time') allows the recommended 


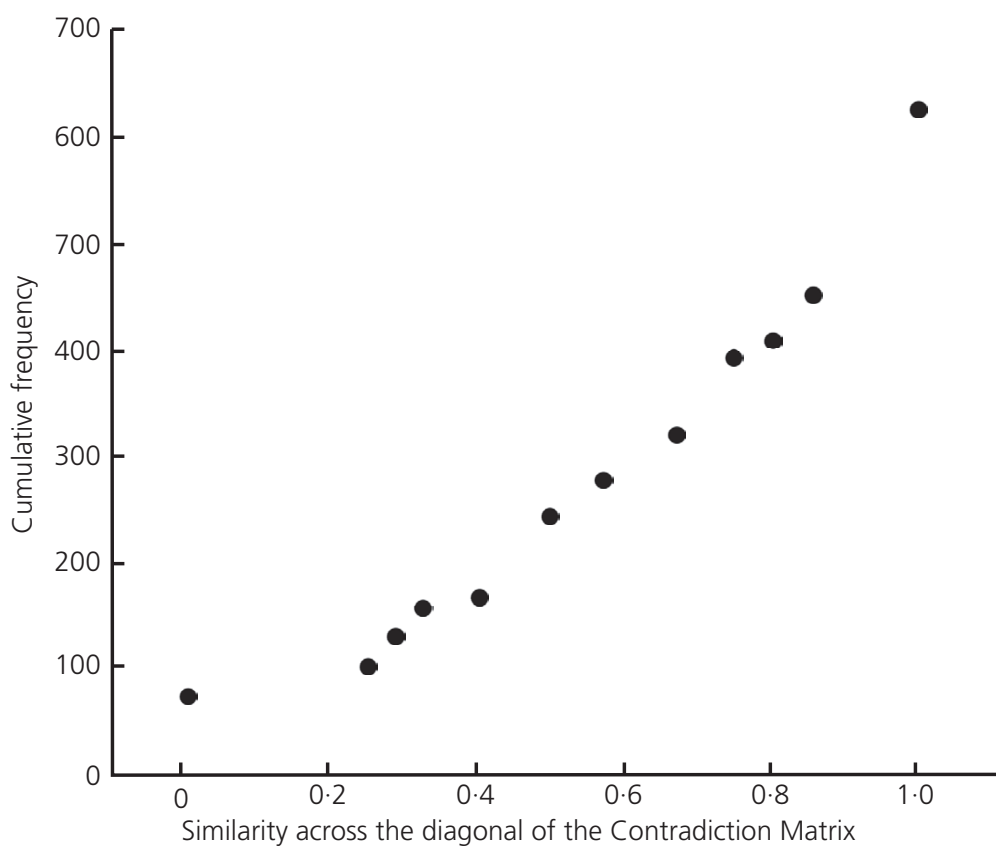

Figure 3. Degree of similarity between the two halves of the TRIZ Contradiction Matrix. Corresponding squares on each side of the diagonal were compared and scored for the similarity of Inventive Principles which they recommended. The total number of principles in each of the two squares was compared with the number which was different. Thus, a score of 1.0 indicates complete identity and 0.5 indicates that half the principles were different. The data are presented as cumulative, with a total of 628 pairs of squares (some of the squares in the matrix are unpopulated) being compared modifications to be expressed in many different ways, so that architecture, food science, management and biology can all be incorporated. Thus, the Contradiction Matrix can be used as a way of categorising trade-offs in biology, with biological principles enumerating the ways in which the trade-offs can be resolved or manipulated. Yet both the parameters and the principles are rooted in engineering. By means of 'trade-off', there is a bridge for biomimetics!

\section{Database or ontology?}

It is easy to store all this information in a database, and to a certain extent this has to happen. Such things as materials, specific trade-offs, principles which solve specific trade-offs, organs and tissues of animals and plants and so on can all be listed. But information is not the same as knowledge. The process of generating useful knowledge starts by creating data from published sources, which can be done automatically using text analysis. It is important to use information from peer-reviewed publications (mostly published in science journals). Sources such as books, television programmes and films are not reliable, yet unwisely they have been used to generate biomimetic data; in fact, even scientific papers are not totally reliable and should be treated with care. The data so created (i.e. collated and verified) can then be assembled into a database, where it becomes information. A database represents the simplest and shortest path to measurable outcomes, but not necessarily to valuable ones. ${ }^{11} \mathrm{~A}$ further problem with a database is that its description of the world is closed. If an item is not present in the database, it is deemed not to exist. The description that an ontology generates assumes an open world. It thus can deal with things which are unknown and can readily accommodate new information. This makes it particularly useful for the description of the understanding of the perceived reality and thus for the description of science.

There is still a gap between information and knowledge. This is crossed using two transformations: abstraction (essentially, removing context) and connectivity (associating items using expressions of logic). ${ }^{11}$ Transformation from information to knowledge depends on the adequacy and correctness of the abstraction methods, rather than mechanical criteria such as the amount of available data or the speed of the search.

There is knowledge now - information in a useful context presented as an ontology.

\section{The ontology}

Ontologies derive in large part from the thinking of philosophers, starting with Aristotle. ${ }^{12}$ In its simplest form, an ontology is a standardised vocabulary. For biologists this is an essential but only partly realised goal. Connectivity of the items in that vocabulary is more easily obtained since computers can be programmed to deal with the logical web of reasoning that the ontologist creates. Both 


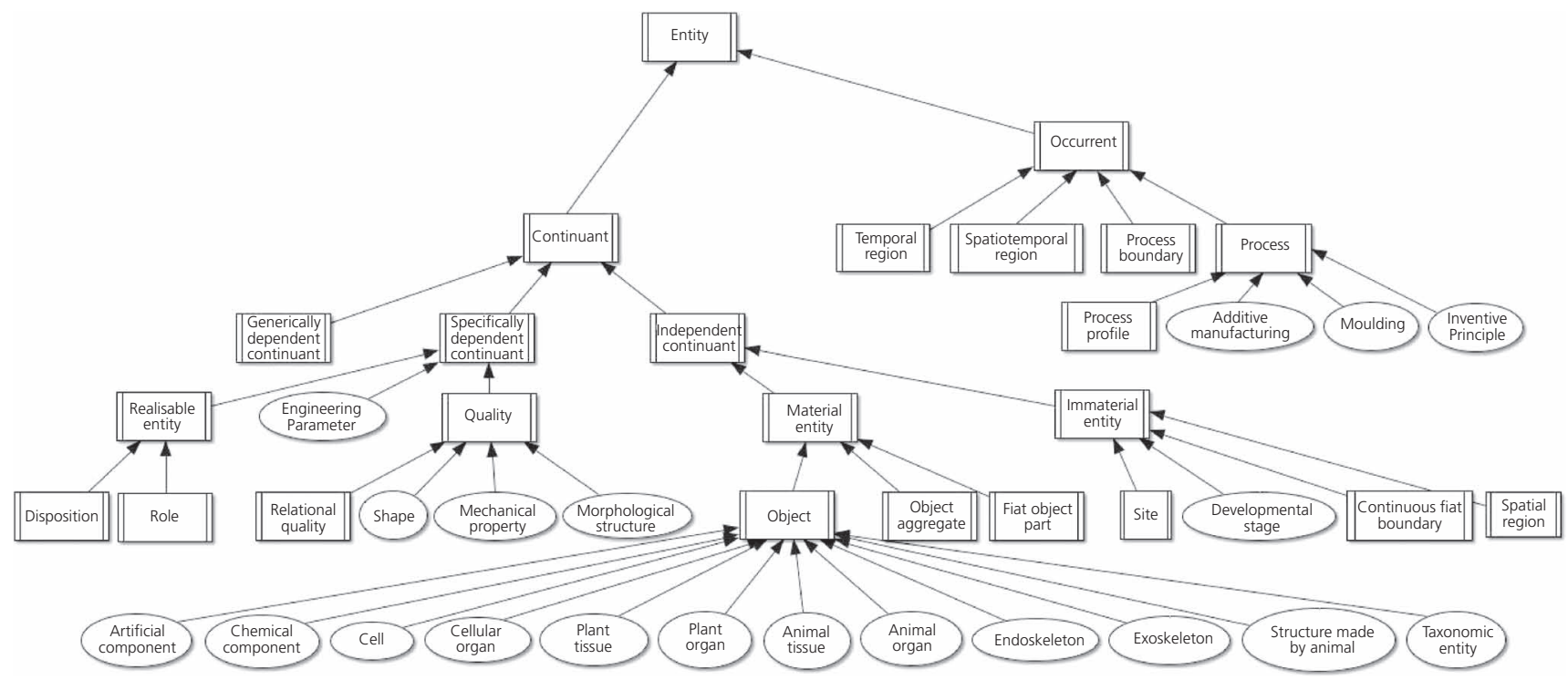

Figure 4. General structure of the ontology. The rectangles represent classes of BFO; the ovals are classes of biomimetic ontology. Only the latter contains information. BFO provides a common framework for ontologies in general. Not all the classes are shown

these requirements emphasise the clear and accurate expression of ideas and objects and their relationships.

The ontology is written in Owl2, the main language of the semantic Web, using the editor Protégé. ${ }^{13}$ It follows the organisation of the Basic Formal Ontology (BFO), ${ }^{12}$ thus ensuring that it can be integrated with other ontologies following the same, widely adopted, format. The BFO (Figure 4) has as its primary classification 'continuants' (things which persist through time) and 'occurrents' (events which occur in time and space). The main continuants are objects, which exist in the absence of any other characteristics. They are therefore independent of those characteristics. However, they have descriptors of one sort or another, such as size, colour, mechanical properties and inbuilt tendencies. These descriptors would not exist without the objects they describe, and so they are dependent continuants. In this ontology, the objects are animals and plants and the things of which they are composed. Although they have a hierarchy (e.g. organisms are made of organs, which are made of tissues, which are in turn made of cells), they are included at a single level within the ontology. Their hierarchical (and other) relationships are expressed by object properties such as is_part_of, is_a and has_part, so that actin is part of muscle, bark cambium is_a meristem and a eukaryotic cell has part nucleus. Other objects included are structures made by animals, chemical components and artificial components and organisms arranged as a formal taxonomy. The taxa have their characteristics (e.g. specific organs such as a shell and sense organs) attached, which adds another layer to the knowledge. The continuants are arranged in classes for ease of handling.
The ontology is based on the concept of the trade-off, which is defined using the method of the TRIZ Contradiction Matrix. The 39 Engineering Parameters are descriptors of the objects, and so they are dependent continuants. They describe and define physical, geometric, positive and negative properties of the objects. They have been modified from the TRIZ originals (which are solely relevant to technology) to give them relevance to biology. Thus, parameter number 31, usually entitled 'harmful side effects', now includes autoimmunity as a possible side effect of the immune system, an essential component of the organism's defence system. Parameter number 39, 'productivity', includes growth, fecundity and rate of foraging.

The ontology in its present condition derives its information from some 400 cases, each one taken from a research paper which defines and solves (at least partially) a trade-off. The cases cover all aspects of biology, from genetics and the molecular structure of materials through to ecosystems and behaviour. The trade-off is commonly defined within this paper as 'trade-off between $[\mathrm{A}]$ and [B]', where A and B can each be identified with one of the Engineering Parameters. This paper will then describe the nature of the trade-off and the mechanism(s) behind its resolution or manipulation. These mechanisms are the means of change or adaptation, and so they are events which occur in time - that is, they are occurrents. In the ontology, they are therefore included in the class 'process' and are derived from the TRIZ list of 40 Inventive Principles. Once again, these principles have been adapted and reformatted to accommodate principles of biological control and change. Thus, principle 26, 'copying', includes reproduction, camouflage and substitution (as when a male spider 
gives the female a faux prey item during courtship, or a cuckoo lays its egg in an alien nest); principle 22, 'convert harm to benefit', includes altruism and sacrificial bonds in tough ceramics. In addition to identifying a trade-off and its resolution, a case is annotated with any taxa which are involved, plus the abstract of the source paper and its origin.

The cases therefore provide the information necessary for the ontology to work. The objects are the biological things or entities which interact with each other and establish the context of the trade-offs; the parameters define the trade-off as the starting point for possible change; and the principles suggest the processes which can lead to resolution or control of the trade-off. Since the cases are not an intrinsic part of the ontology, they are not listed within the BFO schema.

The Inventive Principles also serve as a list of functions that can be assigned to biological objects. Thus, it is possible to identify biological structures that can serve as instances of various functions. The tube foot of an echinoderm is both a deployable structure (principle: dynamism) and a closed hydraulic dynamic effector (principle: pneumatics and hydraulics). Another deployable structure - the proboscis of lepidopterous insects, uses surface-tension forces to move a liquid (surface-tension effect in the principle: counterweight).

\section{Interrogating the ontology}

The ontology is of little use if one cannot extract information from it. Currently, its main use is to deliver ways in which trade-offs such as [allocation/resource], [resolution/sensitivity] and [safety/efficiency] can be resolved. Several Engineering Parameters can be used on each side of the definition of the tradeoff, perhaps in juxtapositions which do not actually occur among the cases providing the information. For example the trade-off [speed/accuracy] can be defined as ((accuracy of measurement) OR (matching prediction) OR (complexity of control) AND ((duration) OR (speed)). The first three parameters concern attributes related to accuracy (was it measured accurately? how close was it to the predicted behaviour? how well controlled is it?), and the second two parameters are related to speed (how long did it take? how quick was it?). Each of these parameters is separately assessed. Use of logical AND ensures

\begin{tabular}{|c|c|c|c|c|}
\hline $\begin{array}{l}\text { Subject } \\
\text { (reference) }\end{array}$ & Detail & Principles & Trade-off A & Trade-off B \\
\hline Ant lion ${ }^{14}$ & Bite prey accurately & $\begin{array}{l}\text { Independent bilateral control } \\
\text { Position feedback }\end{array}$ & Speed of movement & Control of error \\
\hline Archerfish $^{15}$ & Get to prey quickly & $\begin{array}{l}\text { Flexible structure } \\
\text { Ability to predict } \\
\text { Move quickly } \\
\text { Three-dimensional projection }\end{array}$ & Speed of reaction & $\begin{array}{l}\text { Predict where prey } \\
\text { falls }\end{array}$ \\
\hline Bee survival ${ }^{16}$ & $\begin{array}{l}\text { Identify possible } \\
\text { predators }\end{array}$ & $\begin{array}{l}\text { Learning } \\
\text { Take avoiding action }\end{array}$ & Time spent on flower & Control of error \\
\hline Piano player ${ }^{17}$ & $\begin{array}{l}\text { Press the right notes } \\
\text { quickly }\end{array}$ & $\begin{array}{l}\text { Ability to predict } \\
\text { Static-to-dynamic change } \\
\text { Mechanical feedback }\end{array}$ & Control of error & Speed of movement \\
\hline Choose $^{18}$ & $\begin{array}{l}\text { Error related to } \\
\text { learning }\end{array}$ & $\begin{array}{l}\text { Ability to predict } \\
\text { Change response threshold } \\
\text { Error correction } \\
\text { Stochastic accumulator }\end{array}$ & Speed of reaction & Predict outcome \\
\hline Neural $^{19}$ & $\begin{array}{l}\text { Accuracy of visual } \\
\text { search }\end{array}$ & $\begin{array}{l}\text { Change response threshold } \\
\text { Feedback } \\
\text { Stochastic accumulator }\end{array}$ & Control of error & Speed of reaction \\
\hline $\begin{array}{l}\text { Identity of } \\
\text { odour }^{20}\end{array}$ & $\begin{array}{l}\text { Discriminate similar } \\
\text { odours }\end{array}$ & $\begin{array}{l}\text { Network } \\
\text { Feedback }\end{array}$ & Speed of reaction & Predict identity \\
\hline $\begin{array}{l}\text { Vision in } \\
\text { toads }^{21}\end{array}$ & $\begin{array}{l}\text { Discriminate in low } \\
\text { light level }\end{array}$ & $\begin{array}{l}\text { Amplitude change } \\
\text { Change response threshold } \\
\text { Integrate stimuli } \\
\text { Signal-to-noise ratio }\end{array}$ & Speed of reaction & $\begin{array}{l}\text { Difficult } \\
\text { discrimination }\end{array}$ \\
\hline
\end{tabular}

The details summarise the nature of the trade-off, the principles are the subclasses of the Inventive Principles (see Figures 5 and 6 for illustration of this) and the columns 'Trade-off $A$ ' and 'Trade-off $B$ ' are the nature of the speed/accuracy trade-off. More details are in the text

Table 1. Results of querying the ontology for speed/accuracy

trade-offs 
Bioinspired, Biomimetic and Nanobiomaterials Volume 6 Issue BBN2
The trade-off: a central concept for biomimetics

Vincent that both halves of the query have to be present for the response to be triggered. Logical OR allows any of the features so linked to be used in the same way within the query. Thus, this query will present cases where speed and accuracy are traded off in various ways.

Table 1 summarises cases from the ontology which are defined by the [speed/accuracy] trade-off: several studies are highlighted, ranging from behaviour to sensing and neural mechanisms. The column 'Principles' is populated with detailed subclasses developed to be relevant to biology. An example developed from principle 15, 'introduce dynamism', is shown in Figure 5. A brief explanation of dynamism, which appears as an annotation in the ontology, is as follows.

Relax stresses or strains so that shapes or parts find optimal operating conditions and are movable or adaptive rather than rigid or inflexible. A lamp has been made more controllable, introducing hinges. The penalty has been an increasing number of parts. The solution has been further improved by transition to elastic components. The single elastic component has many microlevel parts and very many very small hinges. Here the segmentation principle helps the system to get more dynamic. Generally, if the improvement by one principle causes new difficulties, involve a different principle to solve the new problem. Stiff and immovable structures are often replaced by more dynamic ones: wings that change form and other dynamic structures. The cells which compose organisms, or the structures they produce, may well be able to resist only low loads from the environment and bend out of the way of high loads, or readjust in the presence of (detected) high loads. The nervous system is necessary for detection; the muscular system is necessary for spatial readjustment in the short term, and growth can provide such adjustment in the long term. Evolution and speciation, as the ultimate adaptation mechanism, has to come here.

Therefore, dynamism covers mechanisms of adaptation, and so is one of the principles which appears in six of the eight cases in Table 1, as does the principle 'feedback'. Two other principles, 'consolidation' (Figure 6) and 'preparatory action', also appear several times. Taken together, these four principles provide a strong

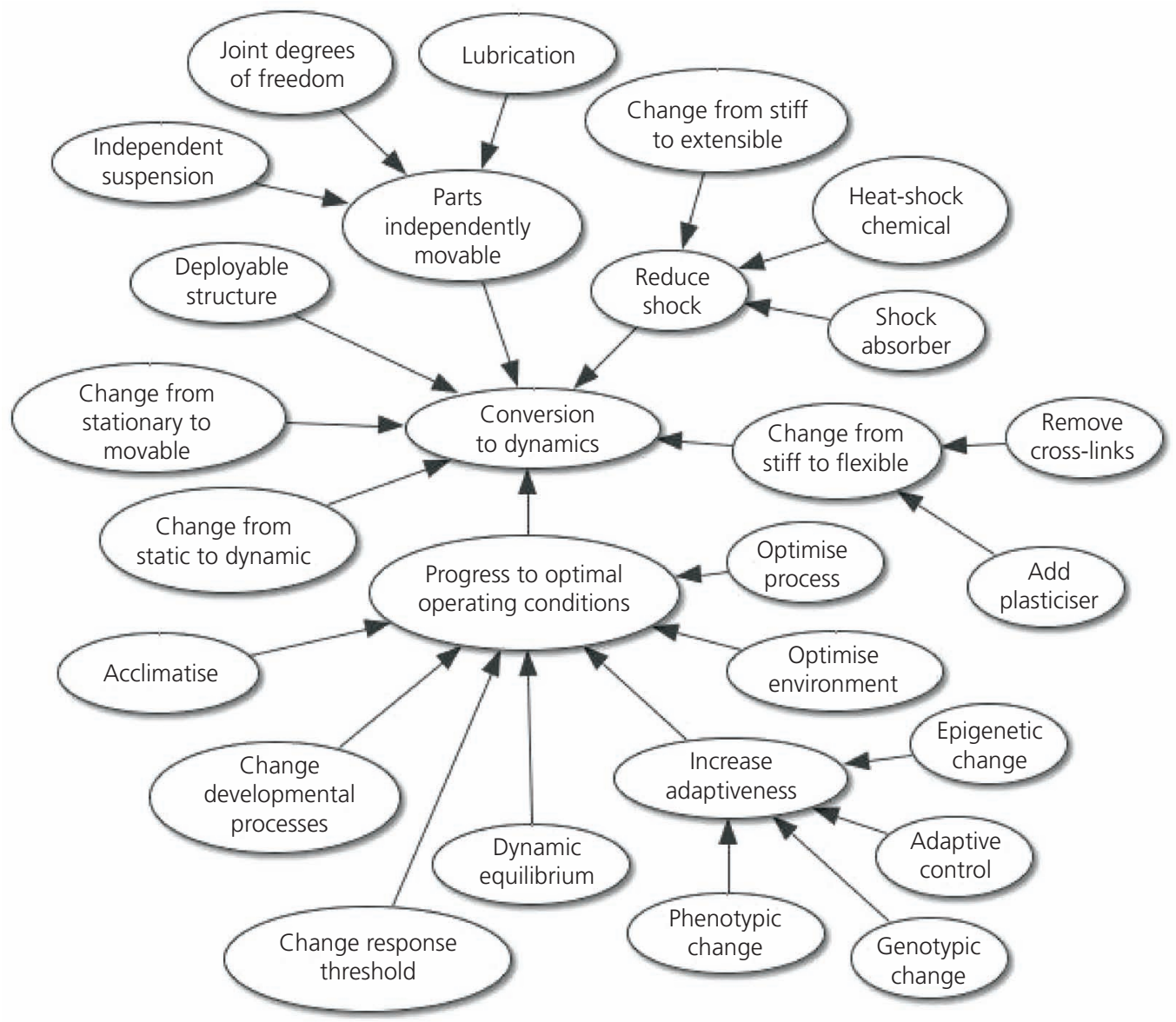

Figure 5. General structure of the Inventive Principle 'conversion to dynamics' to illustrate incorporation of biological terms. Classes lower down the hierarchy are further from the centre 


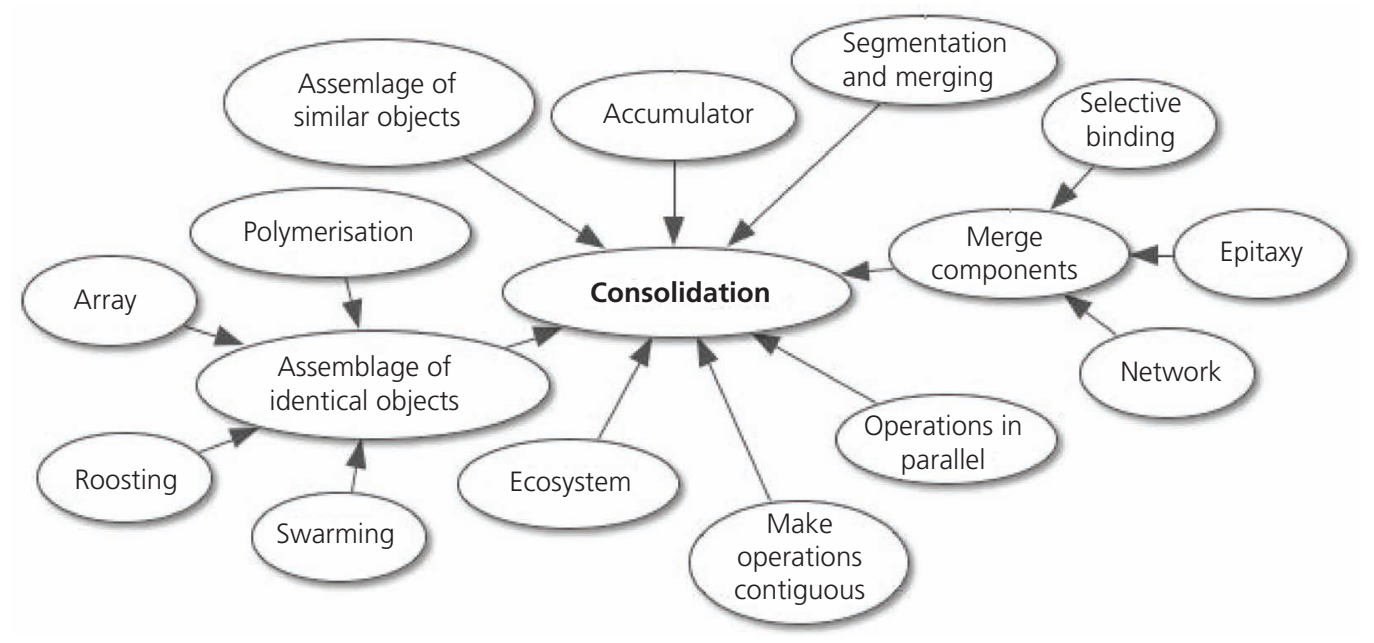

Figure 6. General structure of the Inventive Principle 'Consolidation' to illustrate incorporation of biological terms. Classes lower down the hierarchy are further from the centre

set of suggestions for ways in which a problem involving the tradeoff [speed/accuracy] could be resolved using biomimetic principles.

Another way to use the ontology is to identify biological structures and materials that can perform the various functions indicated by the Inventive Principles. This has the useful feature that although the functions are identified at a detailed level in the cases, they can be summoned by a more general call. Thus, Inventive Principle 15 has a number of subclasses (Figure 5); a query for 'change from stiff to flexible' will return not only materials and structures that can change from stiff to flexible, but also materials that have been identified only by their ability to do this by reducing cross-linking or by incorporating a plasticiser. There is no need to say that they change from stiff to flexible, simply that they perform processes that will lead to that outcome. The ontology thus not only identifies the general means by which an organism copes with, or manipulates, a trade-off, but also itemises the materials and mechanisms that are responsible for this. There is, of course, no reason why artificial materials and structures - and of course biomimetic materials and structures - cannot be included. So the ontology also includes examples where a particular function has already been achieved in technology by a biomimetic route.

\section{Discussion}

An important assumption appears to be that the parameters and principles of TRIZ, although not used directly in their engineering form in this ontology, provide a more or less complete checklist for a reality describing what organisms are and do. The point is not that the checklist is accurate or conclusive, but that such a list can be generated at all. In practice it is not too difficult to assign biological functions to individual elements of this checklist, ensuring that the definitions are moderately well respected so that there is little overlap between the individual classes. This works well with the parameters, which can be declared disjoint within the ontology, showing that as long as there are only two parameters required to define a trade-off for each case, there is no overlap between them. And, anyway, in the majority of the papers, the trade-off is defined objectively by the author, which makes it easy to select an appropriate pair of parameters. Other papers where a trade-off has not been specifically identified have been included because a problem was stated, defined and solved. If the problem could be redefined as a trade-off, the paper was included in the analysis. Many biological papers do not solve a problem - they are descriptive (e.g. papers dealing with morphology and taxonomy, including the taxonomy of ecology) and many papers describe a problem but do not solve it in a clear manner. Such papers were not included although, since they were about biology and therefore about organisms whose evolution has involved trade-offs, they have useful information hidden away.

The trouble starts with the Inventive Principles. The TRIZ Contradiction Matrix, the foundation of this ontology, together with its elements, the parameters and principles, was established with next to no documentation and with questionable objectivity. Hence, much care was taken to analyse each case as objectively as possible before admitting it to the ontology as a source of information. The assessment of the cases was rendered more objective using a text analysis program, Gate (General Architecture for Text Engineering ${ }^{22}$ ). This was mainly used for annotation in conjunction with a gazetteer specially developed for the ontology. This approach highlighted that a wide range of terms and a wide range of styles of reporting are used in biology, both rendering objective analysis more difficult. Disambiguation required assessment of context, although more advanced use of Gate might obviate this. A certain amount of disambiguation is available from within the ontology, since the classes can be tested 
for overlap by both making them disjoint and seeing how the rest of the ontology might be affected by selectively making classes and axioms disjoint and also by careful use of the object properties. Ultimately, there is no reason to retain the classification handed down by TRIZ. Biology is pragmatic, and if some statistical analysis were to show that there are significant gaps or overlap in the TRIZ list of principles, then it would have to be revised. However, such changes would have to be made with great care, since the present list allows biomimetic design to interchange freely with technical design where TRIZ has been freely used. Interestingly, although TRIZ can be used in many contexts, the differences discussed only between engineering TRIZ and biological TRIZ are far less prominent when the biological example is significantly 'engineering', as in the mechanical design of organisms. Some things are constant. But aspects of fracture mechanics - governed largely by considerations of strain energy are very different between the two disciplines. ${ }^{23}$

A further cross-check is available from a pair of graphs which compare the major differences between biology and technology when solving problems. ${ }^{24}$ They show that whereas the success of technology relies on energy, in particular at the micrometre level (i.e. materials processing), the survival of organisms relies on information (derived from the genetic inheritance) and structure (e.g. the molecular shapes used within biological materials, derived from the genetic information). The analysis of the cases shows that the Inventive Principles which make their main recommendations in the origination and use of energy are much less represented in biology than in technology, and those concerned with structure are much better represented in biology than in technology. ${ }^{25}$ Since the two graphs from Vincent et al. $^{24}$ were derived independently of the ontology, this is not only a confirmation of the findings of the ontology, but also a very strong pointer to the general direction in which biomimetics should be moving, at a level as basic as design. The difference found in comparing principles concerned with information is less clear. This is probably because information did not register as an important factor in design in the period when TRIZ was developed, since information could not be processed as readily 40 years ago (when the principles were probably finally established) as it can today with the proliferation of computer modelling. A statistical analysis of the distribution of principles among the various trade-offs may be possible, but even then the sample size, at least of biological cases, is far too small.

Finally, hierarchy: from submolecular scale to ecosystem, biology has 15 to 20 levels of hierarchy. Experience shows that this does not change the solutions to problems which this ontology reveals. Taken on its own, this is strange. But the graph ${ }^{24}$ showing the manipulations required to solve a problem in biology shows very little change in the importance of the six parameters chosen there (namely substance, structure, energy, space, time and information), suggesting that at the level of generalisation of the TRIZ principles, hierarchy is not a factor. The precise mechanisms used in a biological system may well vary with the level of hierarchy, but it is not certain in every instance that the suggested biomimetic solution to a problem will include a biologically derived process in its technical implementation, although the ontology contains such solutions if they are desired.

\section{Conclusion}

This paper describes a work in progress. However, the ontology can already identify trade-offs, suggest biological analogues and the principles which they use and then give more details about the materials, mechanisms and structures that can deliver the functions which the principles suggest, either in a biological context (which can be translated into a technical context by the ontology) or directly in a technical context. To do this, the ontology needs information. It transforms this information into knowledge by generating links between the 'atoms' of information showing causality and correlation, association and separation, process and identity. With enough atoms of information, skilfully linked, the ontology becomes a research tool, yielding insight. Eventually, it will become a means of deriving principles and solutions in biomimetics among systems which have not yet been considered.

A final, Parthian, shot: much of evolution and speciation concerns trade-offs. It is entirely possible that the approach taken by this ontology will generate a succinct account of the interplay of circumstances which lead to speciation and evolution, in particular parallel evolution.

\section{Acknowledgements}

The author is grateful to Marc Desmulliez (Heriot-Watt University) and Marc Weissburg (Georgia Institute of Technology) for their comments on the manuscript.

The ontology is available from the author under Creative Commons license, on condition that it is regarded as a research project and is not to be used commercially without the permission of the author. The author cannot accept responsibility for consequences of its unauthorised use. All modifications to the ontology must be reported to the author.

\section{REFERENCES}

1. Duran-Novoa R, Leon-Rovira N, Aguayo-Tellez H and Said D (2011) Inventive problem solving based on dialectical negation, using evolutionary algorithms and TRIZ heuristic. Computers in Industry 62(4): 437-445.

2. Ashby MF (2013) Materials and the Environment. Elsevier, Oxford, UK.

3. Deb K (2001) Multi-objective Optimization Using Evolutionary Algorithms. Wiley, Chichester, UK.

4. Rolff J and Siva-Jothy MT (2002) Copulation corrupts immunity: a mechanism for a cost of mating in insects. Proceedings of the National Academy of Sciences of the United States of America 99(15): 9916-9918.

5. McKean KA and Nunney L (2001) Increased sexual activity reduces male immune function in Drosophila melanogaster. Proceedings of the National Academy of Sciences of the United States of America 98(14): 7904-7909. 
6. Roff DA and Fairbairn DJ (2007) The evolution of trade-offs: where are we? Journal of Evolutionary Biology 20(2): 433-447.

7. Vlastos G (1982) The Socratic Elenchus. Journal of Philosophy 79(11): 711-714.

8. Popper KR (1963) Conjectures and Refutations. Routledge and Kegan Paul, London, UK.

9. Altshuller G (1988) Creativity as an Exact Science. Gordon \& Breach, New York, NY, USA.

10. Anon (2016) 40 Inventive Principles with Examples. Oxford Creativity, Witney, UK. See https://www.triz.co.uk/files/ U48432_40_inventive_principles_with_examples.pdf (accessed 04/08/2016).

11. Glick J (2013) Ontologies and databases - knowledge engineering for materials informatics. In Informatics for Materials Science and Engineering (Rajan K (ed.)). Butterworth-Heinemann, Oxford, UK, pp. 147-187.

12. Arp R, Smith B and Spear AD (2015) Building Ontologies with Basic Formal Ontology. Massachusetts Institute of Technology, Cambridge, MA, USA.

13. Stanford Center for Biomedical Informatics Research (2016) http://protege.stanford.edu/products.php (accessed 04/08/2106).

14. Lambert EP, Motta PJ and Lowry D (2011) Modulation in the feeding prey capture of the ant-lion, Myrmeleon crudelis. Journal of Experimental Zoology Part A: Ecological Genetics and Physiology 315(10): 602-609.

15. Rossel S, Corlija J and Schuster S (2002) Predicting threedimensional target motion: how archer fish determine where to catch their dislodged prey. Journal of Experimental Biology 205(21): 3321-3326.
16. Ings TC and Chittka L (2008) Speed-accuracy tradeoffs and false alarms in bee responses to cryptic predators. Current Biology 18(19): 1520-1524.

17. Bella SD and Palmer C (2011) Rate effects on timing, key velocity, and finger kinematics in piano performance. $\mathrm{PloS}$ One 6(6): e20518.

18. Goldfarb S, Wong-Lin K, Schwemmer M, Leonard NE and Holmes P (2012) Can post-error dynamics explain sequential reaction time patterns? Frontiers in Psychology 3: 213.

19. Heitz RP and Schall JD (2012) Neural mechanisms of speedaccuracy tradeoff. Neuron 76(3): 616-628.

20. Rinberg D, Koulakov A and Gelperin A (2006) Speedaccuracy tradeoff in olfaction. Neuron 51(3): 351-358.

21. Haldin C, Nymark S, Aho AC, Koskelainen A and Donner K (2009) Rod phototransduction determines the trade-off of temporal integration and speed of vision in dark-adapted toads. Journal of Neuroscience 29(18): 5716-5725.

22. University of Sheffield (2016) https:/gate.ac.uk (accessed 04/ 08/2016).

23. Atkins AG and Mai YW (1985) Elastic and Plastic Fracture: Metals, Polymers, Ceramics, Composites, Biological Materials. Ellis Horwood, Chichester, UK.

24. Vincent JFV, Bogatyreva OA, Bogatyrev NR, Bowyer A and Pahl AK (2006) Biomimetics: its practice and theory. Journal of the Royal Society Interface 3(9): 471-482.

25. Vincent JFV (2014) An ontology of biomimetics. In Biologically Inspired Design: Computational Methods and Tools (Goel AK, McAdams DA and Stone RB (eds)). Springer, New York, NY, USA, pp. 269-285.

\section{HOW CAN YOU CONTRIBUTE? \\ To discuss this paper, please submit up to 500 words to the journal office at journal@ice.org.uk. Your contribution will be forwarded to the author(s) for a reply and, if considered appropriate by the editor-in-chief, it will be published as a discussion in a future issue of the journal.}

ICE Science journals rely entirely on contributions from the field of materials science and engineering. Information about how to submit your paper online is available at www.icevirtuallibrary.com/page/authors, where you will also find detailed author guidelines. 